\title{
Morphological Characterization and Agronomic Evaluation of Yellow Vein Mosaic Virus Resistant Single Cross Hybrids for Yield and Quality Traits in Okra (Abelmoschus esculentus L. Moench)
}

\author{
Shashi Kumar1, Medagam Thirupathi Reddy ${ }^{*}$ \\ ${ }^{1}$ College of Horticulture, Dr. Y.S.R. Horticultural University, Hyderabad, India \\ ${ }^{2}$ Vegetable Research Station, Dr. Y.S.R. Horticultural University, Hyderabad, India \\ Email: "medagamtr@yahoo.co.in
}

Received 5 July 2015; accepted 23 July 2015; published 28 July 2015

Copyright (C) 2015 by authors and OALib.

This work is licensed under the Creative Commons Attribution International License (CC BY). http://creativecommons.org/licenses/by/4.0/

(c) () Open Access

\section{Abstract}

Okra (Abelmoschus esculentus L. Moench) is a traditional pod vegetable widely grown in low, medium and high-input systems for domestic and export markets. The economic and ecological sustainability of okra in these systems is under question because of the low-yielding potential, suboptimal pod quality and susceptibility to biotic and biotic stresses especially the yellow vein mosaic virus (YVMV) of the traditionally cultivated open-pollinated varieties (OPVs) and landraces. Development and use of improved varieties with high yield potential, superior pod quality and resistance to YVMV play an important role in bringing sustainability of okra in these systems. Fifteen single cross hybrids developed by crossing six newly developed inbred lines (RNOYR-14, RNOYR-15, RNOYR-16, RNOYR-17, RNOYR-18 and RNOYR-24), in half-diallel fashion during rainy season 2012, were evaluated along with three local hybrid checks [No. 64 (Mahyco), Avantika (228) (Bioseed) and Shakti (Nunhems)], one OPV check (Arka Anamika) and one OPV cross check for YVMV susceptibility (Pusa Sawani) in a randomized complete block design with three replications during summer 2013 at the Vegetable Research Station, Hyderabad for studying their yield potential, pod quality and resistance to YVMV. All of the 15 single cross hybrids were resistant to YVMV with $0 \%$ incidence as against $\mathbf{1 0 0 \%}$ incidence in OPV checks Arka Anamika and Pusa Sawani. On the basis of mean performance, three crosses RNOYR-15 × RNOYR-16, RNOYR-16 × RNOYR-17 and RNOYR-17 × RNOYR-18 were of significantly higher yield potential than the standard check "No. 64", but of comparable yield potential with other checks "Avantika" and "Shakti". In addition, these three crosses also of superior pod quality could be exploited for development of commercial hybrid okra. These hybrids would be advantageous for production and quality improvement, trade

\footnotetext{
"Corresponding author.
} 


\title{
facilitation and environmental protection.
}

\section{Keywords}

\author{
F1 Hybrids, Inbred Lines, Quality Breeding, Resistance Breeding, Yellow Vein Mosaic Virus
}

\author{
Subject Areas: Ecosystem Science, Plant Science
}

\section{Introduction}

Okra or ladies finger (Abelmoschus esculentus L. Moench) is an important vegetable crop of the Malvaceae family. Okra is said to have originated in tropical Africa [1]. The crop was taken to other parts of the world by the Portuguese [2]. It is widely spread all over tropical, subtropical and warm temperate regions of the world. It is grown in many parts of the world, especially in tropical and sub-tropical countries [3]. It is an important vegetable of the tropical countries and popular among consumers in Cameroon, Ghana, India, Iraq, Nigeria, Pakistan, Switzerland, UAE and USA. Okra has a prominent position among fruit vegetables due to its multiple virtues like high nutritive and medicinal value, ease of cultivation, wide adaptability, year round cultivation, good portability, export potential and bountiful returns [4]. Okra is a diverse crop and it provides possibly more products than any other vegetable crop. It is a multipurpose crop due to its various uses of the fresh leaves, buds, flowers, pods and stems, dry stems, pods and seeds [5]-[8]. It is valued for its edible green seed pods. In the US, Mexico and Japan, the young fruiting pods are the edible portion, while young leaves and mature seeds may be consumed in other countries [9]. It has been used for several purposes [10]. Okra is an important crop from nutritional, medicinal and industrial point of view [11].

Okra is a drought and heat tolerant crop [12]. Being a tropical, hot weather, low land crop and susceptible to low night temperatures, it is extensively cultivated in rainy (June-September) and summer (February-May) seasons in south India [11]. Being an upright, quick growing and medium duration annual herb, it fits well into multiple cropping systems as a either sole crop or intercrop [4]. It is an important cash crop for marginal, small and large farmers, with a potential to boost food, nutritional and health security, foster rural development and support sustainable land care [4]. It has high financial value. It offers a possible route to prosperity for small, medium, and large-scale producers alike [13]. Okra is the object of an intensive production system in urban and rural agriculture [14]. Okra is extensively grown in low, medium and high-input systems by marginal, small and large farmers, respectively who are challenged to produce okra of uniform size and good quality for domestic and export markets. The economic and ecological sustainability of okra in these systems depends primarily on the horticultural superiority and resistance to various biotic and abiotic stresses especially the yellow vein mosaic virus (YVMV). YVMV is affecting okra production for both exporting and domestic consumption in India. Traditionally, low, medium and high-input systems of okra are predominated by the local varieties (landraces). An epidemic of YVMV has devastated okra production based on susceptible landraces. Consequently, a number of open pollinated varieties (OPVs) resistant to this YVMV were developed using traditional breeding approaches, which replaced YVMV susceptible landraces in all these systems. The wild species following interspecific hybridization has introgressed in genes for resistance in the OPVs [15] [16]. Nearly all OPVs of okra traditionally grown in the country are either selections from the landraces or derivatives of pedigree or mutation breeding or hybridization, which are currently being replaced by hybrid varieties ( $\mathrm{F}_{1}$ hybrids). High yielding and YVMV resistant varieties with superior pod quality are able to provide the stable and sustainable production of okra with better acceptance by rural, urban and peri-urban farmers in terms of pod yield and quality.

In India, okra is grown in area of 0.530785 million hectares and has production of 6.350266 million tonnes green pods, whereas productivity of the crop is $12.00 \mathrm{t} / \mathrm{ha}$ [17]. The productivity of okra [17] in India is higher (12.00 tonnes ha ${ }^{-1}$ ) than world average productivity $\left(7.80\right.$ tonnes ha $\left.{ }^{-1}\right)$, but lower than that of Ghana (20 tonnes $\mathrm{ha}^{-1}$ ) and Egypt (14.00 tonnes ha ${ }^{-1}$ ). This is because of low yielding potential of current varieties and reduction in yield due to frequent attacks of pests and diseases, especially the fruit and shoot borer and yellow vein mosaic virus [18]. Further, the economic returns to the okra growers are also low owing to the sub-optimal pod quality [18]. This situation can be improved with genetic improvement through the development and release of im- 
proved varieties having wider genetic base, higher yielding potential, superior pod quality, YVMV resistance and better performance under various agro-climatic conditions. The genetic improvement of okra through breeding has to address and satisfy the needs of both consumers and growers. The objective of okra breeding is to produce better varieties for farmers and growers. Heterosis is the foundation of modern okra breeding programs. Creation of $F_{1}$ hybrids is a key means towards the development of varieties for modern okra production. Hybridization enhances recombination among genes from different strains and selection of desirable types for different environments [19]. Heterosis breeding involving homozygous inbred lines is very important in exploiting heterosis in okra. A number of hybrids resistant/tolerant to YVMV were developed in public and private sectors. Hybrid varieties have more vigor, better quality and yield, and greater disease-resistance than many of the OPVs. Many seed companies in India produce and distribute $F_{1}$ hybrids with high tolerance to YVMV disease that are suitable for the hot and cool season and for export. The use of heterosis breeding for getting high yield with improved quality has already been observed in this crop [20]. Heterosis breeding has lead to the release of several $F_{1}$ hybrids by the private sector [Sobha (Nath Seeds), Sungrow 35 (Sungrow), Vaishali and Vijay (Indo-American Hybrid Seeds), Adhunik and Pancholi (Century Seeds), Hybrid 64 (Mahyco), Azad Kranti (BeejoSheetal), HOK-152 (Syngenta), JK-7135 (JK Seeds), Avantika (228), (Bioseed) and Shakti (Nunhems)] and few hybrids by public sector [HBH-142 (HAU), COBhH 1, Co-3 (Hybrid 8) (TNAU)] have found their way to commercial growers in the country. Even though many studies have been made on heterosis, the pace of work on development of $F_{1}$ hybrids in okra on commercial basis has been limited due to the lack of superior inbred lines in India. Development of agronomically elite inbred lines with good adaptability, good pod quality, good YVMV tolerance, and improved yield potential can provide a genetic base for future hybrid production in India. Considering the potentiality and problems of this crop, there is a prime need for its improvement and to develop improved varieties suitable for specific agro-climatic zones. The final goal of okra breeding programs is then to release new varieties having elite combinations of many desirable horticultural characteristics.

Thus keeping in view the importance of $F_{1}$ hybrids in addressing the aforesaid problems of okra growing, the present study was undertaken 1) to characterize the single cross hybrids on the basis of qualitative traits, 2) to evaluate the mean performance of single cross hybrids for pod yield and its components, 3) to determine the quality of single cross hybrids, 4) to screen the single cross hybrids for resistance to YVMV and 5) to identify the promising varieties for commercial exploitation.

\section{Materials and Methods}

\subsection{Plant Material and Experimental Site}

The experimental material consisted of the fifteen single cross $F_{1}$ hybrids developed by crossing six newly developed, nearly homozygous, horticulturally superior, optimally divergent and YVMV resistant inbred lines of okra (RNOYR-14, RNOYR-15, RNOYR-16, RNOYR-17, RNOYR-18 and RNOYR-24), in half-diallel fashion during rainy season 2012, which were evaluated along with three hybrid checks [No. 64 (Mahyco), Avantika (228), (Bioseed) and Shakti (Nunhems)] and two OPV checks (Pusa Sawani and Arka Anamika) during summer 2013 at the Vegetable Research Station, Hyderabad for studying their yield potential, pod quality and resistance to YVMV. Hyderabad comes under semi arid tropics with a tropical wet and dry climate.

For comparison of horticultural superiority, the above commercial hybrid checks were included as crosschecks. For screening against YVMV, the above commercial OPV checks which were YVMV resistant when developed but highly susceptible to YVMV at present were included as cross-checks. The Experimental Farm is situated at $17.19^{\circ} \mathrm{N}, 79.23^{\circ} \mathrm{E}$ and $542.6 \mathrm{~m}$ above mean sea level. The soils are sandy loams.

\subsection{Experimental Design and Crop Management}

The experimental design used for this study is the randomized block design. The number of replications was three. Each block was subdivided into 26 plots corresponding to the 26 entries of okra studied. Each entry was grown in a double-row plot. The unit plot size was $3.0 \times 1.2 \mathrm{~m}$. The plants were spaced $60 \mathrm{~cm}$ apart between rows and $30 \mathrm{~cm}$ apart between plants. Each row had 10 plants, while each plot and entry had 20 plants. All agronomic practices were kept constant for whole of the experiment. Recommended package of practices was followed to raise a successful crop. Regular plant protection measures were carried out to safeguard the crop 
from pests and diseases.

\subsection{Recording of Qualitative and Biometric Traits}

The data on 18 qualitative characters based on descriptors for Abelmoschus esculentus [21] were recorded in each entry on a single plant basis. Biometric observations were recorded on five randomly selected competitive plants in each entry in each replication for plant height $(\mathrm{cm})$, number of branches per plant, internodal length (cm), days to 50\% flowering, first flowering node, first fruiting node, fruit length $(\mathrm{cm})$, fruit width $(\mathrm{cm})$, and fruit weight (g) and on whole plot basis for days to $50 \%$ flowering, total number of fruits per plant, number of marketable fruits per plant, total yield per plant (g) and marketable yield per plant (g). Plant height, number of branches per plant and internodal length were all determined at the end of the growing season upon the cessation of effective plant growth. With the help of a decameter ribbon and slide calipers, the height of the plants and length of the internodes were measured. Height of the plant $(\mathrm{cm})$ from ground level to tip of the main stem was measured in centimeters at the time of final harvest. Number of primary branches on the main stem was counted. Length of main stem from the basal node to the last node of green, tender and marketable fruit was measured in centimeters and divided with the number of nodes on the main stem up to the last node of green, tender and marketable fruit to get internodal length. The day when $50 \%$ of the plants flowered in a plot was recorded as the number of days from sowing to flowering. The node on the main stem from ground level at which the first flower appeared was counted. The node on the main stem from ground level at which the first fruit set was counted. Ten tagged pods were harvested 7 days after anthesis early in the morning and immediately carried to the laboratory and average length, width and weight of pods in each entry were determined. Fruit length (cm) was measured as the distance from the fruit cap scar at the base excluding peduncle to the tip end of the pod [22]. Fruit diameter $(\mathrm{cm})$ in the peduncle insertion zone was measured using a standard graduated scale vernier calliper [22]. Fruit weight (g) of okra fruits was determined by weighing fruits individually in a digital analytical balance $( \pm 0.001 \mathrm{~g}$ ). Pods were harvested three times per week, and number and weight of all pods were determined at each harvest. Data from each harvest were combined at the end of the growing season to help estimate of total number of pods per plant and total yield per plant. Total number of green and tender fruits including YVMV and borer infested ones of each plot was recorded under full range of pickings and was averaged by dividing with the number of plants to get number of fruits per plant at fruiting stage. Total number of green and tender fruits excluding YVMV and borer infested ones of each plot was recorded under full range of pickings and was averaged by dividing with the number of plants to get number of marketable fruits per plant at fruiting stage. Total weight of green and tender fruits including YVMV and borer infested ones of each plot was recorded under full range of pickings was summed and averaged by dividing with the number of plants to get total yield per plant. Total weight of green and tender fruits excluding YVMV and borer infested ones of each plot was recorded under full range of pickings, summed and averaged by dividing with the number of plants to get the marketable yield per plant. The incidence of YVMV on plants of each of test entries was recorded at final harvest.

\subsection{Analysis of Biometric Data}

The mean performance of parents, hybrids and checks regarding pod yield and its components was worked out. The data recorded for different characters were analyzed for variance in accordance with the standard technique [23]. Means were compared by the least significant difference (LSD) test, at a significance level of $P \leq 0.05$ and $P \leq 0.01$. The mean replicated values of YVMV infestation on plants were subjected to square root transformation to restore the distribution to normality. The disease reaction was determined as per the scale for classifying disease reaction of okra to yellow vein mosaic virus. The disease reaction was categorized as resistant, tolerant, susceptible and highly susceptible as per the disease severity range of $0 \%, 1 \%-30,31 \%-70 \%$ and $71 \%-100 \%$, respectively [24].

\section{Results}

\subsection{Evaluation of $F_{1}$ Hybrids for Agro-Economic Traits}

\subsubsection{Analysis of Variance}

The analysis of variance (Table 1$)$ showed highly significant differences $(P \leq 0.05)$ among 26 entries involving 
fifteen crosses, their six parents, three hybrid checks and two OPV checks for almost all the traits expect days to 50 per cent flowering and FSB infestation on fruits. No significant differences were observed within replications.

\subsubsection{Average Performance of Hybrids in Comparison to Parents and Checks}

The range of mean values of parents as a group (Table 2) was highest for total yield per plant (274.82 to 335.66

Table 1. Analysis of variance for various agro-economic traits in okra.

\begin{tabular}{lccc}
\hline \multirow{2}{*}{ Character } & \multicolumn{3}{c}{ Sum of squares } \\
\cline { 2 - 4 } & Replications (2) & Treatments (25) & Error (50) \\
\hline Plant height (cm) & 22.783 & $14437.013^{* *}$ & 4149.666 \\
Number of branches per plant & 0.021 & $11.125^{* *}$ & 1.588 \\
Internodal length (cm) & 0.024 & $20.148^{* *}$ & 2.149 \\
Days to 50\% flowering & 1.719 & 14.158 & 23.613 \\
First flowering node & 0.306 & $4.622^{*}$ & 5.026 \\
First fruiting node & 0.306 & $4.622^{*}$ & 5.026 \\
Fruit length (cm) & 0.219 & $41.220^{* *}$ & 21.990 \\
Fruit width (cm) & 0.002 & $0.336^{*}$ & 0.247 \\
Fruit weight (g) & 0.641 & $121.132^{*}$ & 104.066 \\
Total number of fruits per plant & 13.422 & $1493.094^{*}$ & 375.267 \\
Number of marketable fruits per plant & 17.454 & $1811.981^{* *}$ & 442.928 \\
Total yield per plant (g) & 2785.125 & $471639.302^{* *}$ & 128951.109 \\
Marketable yield per plant (g) & 3635.293 & $534393.169^{* *}$ & 117778.651 \\
\hline
\end{tabular}

*, ** Significant at $P=0.05$ and 0.01 levels, respectively; values in parenthesis denote degrees of freedom.

Table 2. Average performance of parental lines, half-diallel crosses and checks in okra.

\begin{tabular}{lcccc}
\hline \multirow{2}{*}{ Character } & \multicolumn{3}{c}{ Average performance } \\
\cline { 2 - 4 } & Parental lines & Cross combinations & Hybrid checks & OPV checks \\
Plant height (cm) & 114.83 & 122.21 & 129.78 & 131.79 \\
Number of branches per plant & 1.48 & 1.58 & 2.04 & 1.74 \\
Internodal length (cm) & 3.74 & 4.05 & 4.33 & 5.30 \\
Days to 50\% flowering & 40.28 & 40.24 & 39.89 & 39.67 \\
First flowering node & 4.83 & 4.91 & 4.78 & 5.00 \\
First fruiting node & 4.83 & 4.91 & 4.78 & 5.00 \\
Fruit length (cm) & 13.47 & 14.10 & 14.68 & 13.02 \\
Fruit width (cm) & 1.73 & 1.70 & 1.73 & 1.74 \\
Fruit weight (g) & 16.80 & 17.76 & 18.91 & 15.91 \\
Total number of fruits per plant & 23.73 & 28.69 & 33.19 & 24.27 \\
Number of marketable fruits per plant & 19.96 & 25.31 & 29.51 & 15.99 \\
Total yield per plant (g) & 298.48 & 385.99 & 473.83 & 291.64 \\
Marketable yield per plant (g) & 250.79 & 341.97 & 428.27 & 191.92 \\
YVMV infestation on plants (\%) & 0.00 & 0.00 & 2.11 & 100.00 \\
\hline
\end{tabular}

YVMV = Yellow vein mosaic virus; OPV = Open pollinate variety. 
g) followed by marketable yield per plant (226.55 to $291.46 \mathrm{~g})$ and plant height (101.93 to $128.90 \mathrm{~cm})$. The range of mean values of crosses as a group was highest for total yield per plant (286.65 to $525.65 \mathrm{~g}$ ) followed by marketable yield per plant (236.65 to $483.35 \mathrm{~g}$ ) and plant height (94.09 to $149.67 \mathrm{~cm}$ ). Single cross hybrids and their parental lines had 0\% incidence of YVMV. Susceptible national OPV checks had 100\%, while hybrid local hybrid checks had $2.11 \%$ incidence of YVMV.

\subsubsection{Mean Performance of Single Cross Hybrids}

Mean performance of the growth attributes is presented in Table 3. Plant height among the parents and hybrids

Table 3. Mean performance of hybrids in comparison to their parents and hybrid checks for growth and earliness attributes of okra.

\begin{tabular}{|c|c|c|c|c|c|c|}
\hline Entry & $\begin{array}{l}\text { Plant height } \\
\text { (cm) }\end{array}$ & $\begin{array}{c}\text { Number } \\
\text { branches per plant }\end{array}$ & $\begin{array}{l}\text { Internodal length } \\
\text { (cm) }\end{array}$ & $\begin{array}{c}\text { Days to } \\
50 \% \text { flowering }\end{array}$ & $\begin{array}{c}\text { First } \\
\text { flowering node }\end{array}$ & $\begin{array}{c}\text { First } \\
\text { fruiting node }\end{array}$ \\
\hline \multicolumn{7}{|l|}{ Single cross hybrids } \\
\hline RNOYR-14 × RNOYR-15 & 120.87 & 1.47 & 3.81 & 40.00 & 5.00 & 5.00 \\
\hline RNOYR-14 × RNOYR-16 & 123.11 & 1.73 & 4.47 & 40.00 & 4.67 & 4.67 \\
\hline RNOYR-14 × RNOYR-17 & 115.09 & 1.33 & 4.04 & 39.67 & 4.33 & 4.33 \\
\hline RNOYR-14 × RNOYR-18 & 106.67 & 1.20 & 3.57 & 40.33 & 5.00 & 5.00 \\
\hline RNOYR-14 × RNOYR-24 & 93.18 & 1.70 & 3.17 & 40.33 & 5.00 & 5.00 \\
\hline RNOYR-15 × RNOYR-16 & 149.67 & 2.13 & 4.47 & 40.00 & 5.00 & 5.00 \\
\hline RNOYR-15 × RNOYR-17 & 128.20 & 1.47 & 4.35 & 40.33 & 5.33 & 5.33 \\
\hline RNOYR-15 × RNOYR-18 & 126.27 & 1.60 & 4.17 & 40.33 & 5.00 & 5.00 \\
\hline RNOYR-15 × RNOYR-24 & 131.79 & 1.40 & 4.20 & 40.33 & 5.00 & 5.00 \\
\hline RNOYR-16 × RNOYR-17 & 145.63 & 1.93 & 4.69 & 40.33 & 4.33 & 4.33 \\
\hline RNOYR-16 × RNOYR-18 & 124.77 & 1.87 & 4.14 & 40.67 & 5.00 & 5.00 \\
\hline RNOYR-16 × RNOYR-24 & 112.07 & 1.40 & 3.87 & 40.33 & 5.00 & 5.00 \\
\hline RNOYR-17 × RNOYR-18 & 135.73 & 1.47 & 4.07 & 40.00 & 5.00 & 5.00 \\
\hline RNOYR-17 × RNOYR-24 & 125.95 & 1.73 & 4.32 & 40.67 & 5.00 & 5.00 \\
\hline RNOYR-18 × RNOYR-24 & 94.09 & 1.20 & 3.47 & 40.33 & 5.00 & 5.00 \\
\hline \multicolumn{7}{|l|}{ Parental inbred lines } \\
\hline RNOYR-14 & 114.60 & 1.57 & 3.52 & 40.00 & 5.00 & 5.00 \\
\hline RNOYR-15 & 128.90 & 1.10 & 3.77 & 40.67 & 4.67 & 4.67 \\
\hline RNOYR-16 & 119.77 & 2.37 & 3.88 & 40.17 & 4.33 & 4.33 \\
\hline RNOYR-17 & 113.53 & 0.90 & 3.42 & 41.17 & 5.00 & 5.00 \\
\hline RNOYR-18 & 101.93 & 1.00 & 3.61 & 40.00 & 5.00 & 5.00 \\
\hline RNOYR-24 & 110.27 & 1.93 & 4.23 & 39.67 & 5.00 & 5.00 \\
\hline \multicolumn{7}{|l|}{ Hybrid checks } \\
\hline No. 64 & 126.19 & 2.33 & 4.44 & 40.33 & 4.67 & 4.67 \\
\hline Avantika & 129.19 & 2.13 & 4.27 & 39.67 & 5.00 & 5.00 \\
\hline Shakti & 133.95 & 1.67 & 4.29 & 39.67 & 4.67 & 4.67 \\
\hline \multicolumn{7}{|l|}{ OPV checks } \\
\hline Pusa Sawani & 133.75 & 1.83 & 5.42 & 39.00 & 5.00 & 5.00 \\
\hline Arka Anamika & 129.82 & 1.64 & 5.18 & 40.33 & 5.00 & 5.00 \\
\hline CV (\%) & 7.46 & 10.99 & 5.04 & 1.71 & 6.50 & 6.50 \\
\hline CD (5\%) & 14.95 & 0.29 & 0.34 & NS & 0.52 & 0.52 \\
\hline
\end{tabular}

OPV = Open pollinate variety. 
ranged from $101.93 \mathrm{~cm}$ (RNOYR-18) to $128.90 \mathrm{~cm}$ (RNOYR-15) and $93.18 \mathrm{~cm}$ (RNOYR-14 $\times$ RNOYR-24) to $149.67 \mathrm{~cm}$ (RNOYR-15 $\times$ RNOYR-16), respectively. The hybrid RNOYR-15 $\times$ RNOYR-16 (149.67 cm) was significantly taller than all the three hybrid checks while RNOYR-16 $\times$ RNOYR-17 $(145.63 \mathrm{~cm})$ was significantly taller than the "No. 64" and "Avantika". None of the parents were significantly taller than the three hybrid checks. Number of branches per plant varied from $0.90 \mathrm{~cm}$ (RNOYR-17) to $2.37 \mathrm{~cm}$ (RNOYR-16) and 1.20 cm (RNOYR-18 $\times$ RNOYR-24) to $2.13 \mathrm{~cm}$ (RNOYR-15 $\times$ RNOYR-16) among the parents and hybrids, respectively. The hybrids RNOYR-15 × RNOYR-16 $(2.13 \mathrm{~cm})$ and RNOYR-16 $\times$ RNOYR-17 $(1.93 \mathrm{~cm})$ had significantly higher number of branches per plant than "Shakti". The parents RNOYR-16 and RNOYR-24 had significantly more number of branches per plant than "Avantika" and "Shakti", respectively. Internodal length varied from 3.42 (RNOYR-17) to 4.23 (RNOYR-24) among the parents and 3.17 (RNOYR-14 × RNOYR-24) to 4.69 (RNOYR-16 $\times$ RNOYR-17) among the hybrids. Among 15 hybrids, five hybrids RNOYR-14 $\times$ RNOYR-15 (3.81), RNOYR-14 × RNOYR-18 (3.57), RNOYR-14 × RNOYR-24 (3.17), RNOYR-16 × RNOYR-24 (3.87) and RNOYR-18 × RNOYR-24 (3.47) had significantly shorter internodal length than all the three hybrid checks. Almost all parents expect RNOYR-24 had significantly shorter internodal length than all the three hybrid checks.

The parents, their hybrids, hybrid checks and OPV checks did not differ significantly for days to $50 \%$ flowering (Table 3). Mean performance of the earliness attributes is presented in Table 3. First flowering and fruiting node in the parents and hybrids ranged from 4.33 (RNOYR-16) to 5.00 (RNOYR-17, RNOYR-18 and RNOYR24) and 4.33 (RNOYR-14 × RNOYR-17 and RNOYR-16 × RNOYR-17) to 5.33 (RNOYR-15 × RNOYR-17), respectively. Two hybrids namely RNOYR-14 × RNOYR-17 (4.33) and RNOYR-16 × RNOYR-17 (4.33) and one parent RNOYR-16 (4.33) had produced their first flower and fruit at the significantly lower node than the check "Avantika".

Mean performance of the fruit attributes is presented in Table 4. Fruit length of the parents and hybrids varied from $12.59 \mathrm{~cm}$ (RNOYR-17) to $14.37 \mathrm{~cm}$ (RNOYR-14) and $12.67 \mathrm{~cm}$ (RNOYR-18 × RNOYR-24) to $14.85 \mathrm{~cm}$ (RNOYR-17 $\times$ RNOYR-18), respectively. None of the parents and hybrids had significantly longer fruits than all the three checks. Fruit width of the parents and hybrids ranged from $1.64 \mathrm{~cm}$ (RNOYR-24) to $1.81 \mathrm{~cm}$ (RNOYR-18) and $1.58 \mathrm{~cm}$ (RNOYR-14 $\times$ RNOYR-15 and RNOYR-14 $\times$ RNOYR-17) to $1.84 \mathrm{~cm}$ (RNOYR-15 $\times$ RNOYR-18), respectively.

None of the parents and hybrids had significantly wider fruits than all the three checks. Fruit weight of the parents and hybrids varied from $15.69 \mathrm{~g}$ (RNOYR-24) to $18.20 \mathrm{~g}$ (RNOYR-18) and $15.41 \mathrm{~g}$ (RNOYR-14 $\times$ RNOYR-17) to 19.76 g (RNOYR-15 $\times$ RNOYR-18), respectively. None of the parents and hybrids had significantly heavier fruits than any of the three hybrid checks. Total number of fruits produced by the parents and hybrids ranged from 20.73 (RNOYR-18) to 27.67 (RNOYR-16) and 23.09 (RNOYR-18 $\times$ RNOYR-24) to 37.12 (RNOYR-15 $\times$ RNOYR-16), respectively. The hybrid RNOYR-15 $\times$ RNOYR-16 produced significantly more total number of fruits per plant than the check No. 64 (36.42). Number of marketable fruits produced by the parents and hybrids ranged from 16.73 (RNOYR-18) to 24.00 (RNOYR-16) and 19.09 (RNOYR-18 $\times$ RNOYR-24) to 33.79 (RNOYR-15 × RNOYR-16), respectively. Only one hybrid RNOYR-15 × RNOYR-16 (33.79) had produced significantly more number of marketable fruits per plant than the check "No. 64" (27.77 g).

Mean performance of the entries for yield attributes is presented in Table 4. Total yield per plant produced by the parents and hybrids ranged from $274.82 \mathrm{~g}$ (RNOYR-24) to $335.66 \mathrm{~g}$ (RNOYR-16) and $286.29 \mathrm{~g}$ (RNOYR$18 \times$ RNOYR-24) to 525.65 g (RNOYR-15 $\times$ RNOYR-16), respectively. Only one hybrid RNOYR-15 $\times$ RNOYR-16 had produced significantly higher total yield per plant (525.65 g) than the check "No. 64". Marketable yield per plant produced by the parents and hybrids varied from $226.55 \mathrm{~g}$ (RNOYR-18) to $291.46 \mathrm{~g}$ (RNOYR-16) and 236.65 g (RNOYR-18 × RNOYR-24) to 483.35 g (RNOYR-15 × RNOYR-16), respectively. Only one hybrid RNOYR-15 × RNOYR-16 produced significantly higher marketable yield per plant (483.35 g) than the check "No. 64".

\subsection{Characterization of Single Cross Hybrids for Qualitative Traits}

Fifteen half-diallel crosses characterized in this study showed a broad variation for most traits (Table 5). All of the fifteen crosses and their parents, three hybrid checks and two OPV checks had the commonest growth habit i.e. indeterminate growth habit. There were no differences in mature leaf color (only green) and leaf rib colour (only green) among okra hybrids. Petiole colour among the hybrids varied from green to green with red colour. Hybrids were grouped into two groups on the basis of their leaf shape (shallowly lobed and deeply lobed) and 
Table 4. Mean performance of hybrids in comparison to their parents and hybrid checks for fruit and yield attributes of okra.

\begin{tabular}{|c|c|c|c|c|c|c|c|c|}
\hline Entry & $\begin{array}{c}\text { Fruit } \\
\text { length } \\
(\mathrm{cm})\end{array}$ & $\begin{array}{c}\text { Fruit } \\
\text { width } \\
\text { (cm) }\end{array}$ & $\begin{array}{l}\text { Fruit } \\
\text { weight } \\
\text { (g) }\end{array}$ & $\begin{array}{c}\text { Total no. of } \\
\text { fruits per } \\
\text { plant }\end{array}$ & $\begin{array}{c}\text { No. of } \\
\text { marketable } \\
\text { fruits per plant }\end{array}$ & $\begin{array}{l}\text { Total yield } \\
\text { per plant } \\
\text { (g) }\end{array}$ & $\begin{array}{l}\text { Marketable } \\
\text { yield per plant } \\
\text { (g) }\end{array}$ & $\begin{array}{c}\text { YVMV } \\
\text { infestation } \\
(\%)\end{array}$ \\
\hline \multicolumn{9}{|l|}{ Single cross hybrids } \\
\hline RNOYR-14 × RNOYR-15 & 14.61 & 1.58 & 17.98 & 29.08 & 25.75 & 392.63 & 347.61 & $0.00(\mathrm{R})$ \\
\hline RNOYR-14 × RNOYR-16 & 13.99 & 1.68 & 17.26 & 26.57 & 23.57 & 343.36 & 304.91 & $0.00(\mathrm{R})$ \\
\hline RNOYR-14 × RNOYR-17 & 14.23 & 1.58 & 15.41 & 25.85 & 22.51 & 298.88 & 260.92 & $0.00(\mathrm{R})$ \\
\hline RNOYR-14 × RNOYR-18 & 13.45 & 1.77 & 17.52 & 25.91 & 22.58 & 340.68 & 297.19 & $0.00(\mathrm{R})$ \\
\hline RNOYR-14 × RNOYR-24 & 13.89 & 1.72 & 18.28 & 27.84 & 24.18 & 381.95 & 332.15 & $0.00(\mathrm{R})$ \\
\hline RNOYR-15 × RNOYR-16 & 14.37 & 1.77 & 18.64 & 37.12 & 33.79 & 525.65 & 483.35 & $0.00(\mathrm{R})$ \\
\hline RNOYR-15 × RNOYR-17 & 13.33 & 1.71 & 16.09 & 26.51 & 23.17 & 318.67 & 278.20 & $0.00(\mathrm{R})$ \\
\hline RNOYR-15 × RNOYR-18 & 14.61 & 1.84 & 19.76 & 28.17 & 25.17 & 416.29 & 371.43 & $0.00(\mathrm{R})$ \\
\hline RNOYR-15 × RNOYR-24 & 14.82 & 1.69 & 17.65 & 28.34 & 25.34 & 375.05 & 335.44 & $0.00(\mathrm{R})$ \\
\hline RNOYR-16 × RNOYR-17 & 14.72 & 1.70 & 18.63 & 35.41 & 31.75 & 495.22 & 450.90 & $0.00(\mathrm{R})$ \\
\hline RNOYR-16 × RNOYR-18 & 13.59 & 1.73 & 17.22 & 29.49 & 26.16 & 379.39 & 337.13 & $0.00(\mathrm{R})$ \\
\hline RNOYR-16 × RNOYR-24 & 14.07 & 1.71 & 18.99 & 25.61 & 22.28 & 365.79 & 318.60 & $0.00(\mathrm{R})$ \\
\hline RNOYR-17 × RNOYR-18 & 14.85 & 1.68 & 18.21 & 33.62 & 29.95 & 489.90 & 440.60 & $0.00(\mathrm{R})$ \\
\hline RNOYR-17 × RNOYR-24 & 14.33 & 1.70 & 18.25 & 27.77 & 24.43 & 380.07 & 334.50 & $0.00(\mathrm{R})$ \\
\hline RNOYR-18 × RNOYR-24 & 12.67 & 1.72 & 16.51 & 23.09 & 19.09 & 286.29 & 236.65 & $0.00(\mathrm{R})$ \\
\hline \multicolumn{9}{|l|}{ Parental inbred lines } \\
\hline RNOYR-14 & 14.37 & 1.69 & 17.23 & 23.33 & 19.67 & 301.81 & 254.26 & $0.00(\mathrm{R})$ \\
\hline RNOYR-15 & 13.37 & 1.68 & 15.85 & 24.73 & 20.73 & 295.06 & 247.96 & $0.00(\mathrm{R})$ \\
\hline RNOYR-16 & 13.83 & 1.74 & 16.15 & 27.67 & 24.00 & 335.66 & 291.46 & $0.00(\mathrm{R})$ \\
\hline RNOYR-17 & 12.59 & 1.80 & 17.68 & 22.67 & 19.33 & 301.70 & 257.02 & $0.00(\mathrm{R})$ \\
\hline RNOYR-18 & 13.03 & 1.81 & 18.20 & 20.73 & 16.73 & 281.85 & 226.55 & $0.00(\mathrm{R})$ \\
\hline RNOYR-24 & 13.63 & 1.64 & 15.69 & 23.27 & 19.27 & 274.82 & 227.50 & $0.00(\mathrm{R})$ \\
\hline \multicolumn{9}{|l|}{ Hybrid checks } \\
\hline No. 64 & 14.37 & 1.73 & 18.19 & 31.43 & 27.77 & 429.16 & 400.88 & $0.00(\mathrm{R})$ \\
\hline Avantika & 15.01 & 1.73 & 19.35 & 33.10 & 30.10 & 482.04 & 438.20 & $8.33(\mathrm{~T})$ \\
\hline Shakti & 14.67 & 1.74 & 19.20 & 35.03 & 30.66 & 510.29 & 445.73 & $8.33(\mathrm{~T})$ \\
\hline \multicolumn{9}{|l|}{ OPV checks } \\
\hline Pusa Sawani & 12.31 & 1.85 & 16.53 & 20.27 & 12.87 & 254.77 & 161.63 & 100.00 (HS) \\
\hline Arka Anamika & 13.73 & 1.63 & 15.28 & 28.27 & 19.10 & 328.50 & 222.20 & 100.00 (HS) \\
\hline CV (\%) & 4.75 & 4.09 & 8.23 & 9.89 & 12.49 & 13.78 & 15.19 & - \\
\hline CD (5\%) & 1.08 & 0.11 & 2.36 & 4.50 & 4.88 & 83.30 & 79.62 & - \\
\hline
\end{tabular}

OPV = Open pollinate variety; Values in parenthesis denote YVMV disease reaction; YVMV = Yellow vein mosaic virus; HS = Highly susceptible; $\mathrm{R}=$ Resistant; $\mathrm{T}=$ Tolerant 
Table 5. Qualitative traits of single cross hybrids in comparison to standard checks of okra.

(a)

\begin{tabular}{|c|c|c|c|c|c|c|}
\hline$F_{1}$ hybrid & $\begin{array}{c}\text { Plant } \\
\text { growth } \\
\text { habit }\end{array}$ & $\begin{array}{c}\text { Branching } \\
\text { habit }\end{array}$ & Stem colour & Stem pubescence & $\begin{array}{l}\text { Mature } \\
\text { leaf } \\
\text { colour }\end{array}$ & $\begin{array}{c}\text { Leaf rib } \\
\text { colour }\end{array}$ \\
\hline \multicolumn{7}{|l|}{ Single cross hybrids } \\
\hline RNOYR-14 × RNOYR-15 & Erect & Low & Green & Smooth & Green & Green \\
\hline RNOYR-14 × RNOYR-16 & Erect & Low & Green & Smooth & Green & Green \\
\hline RNOYR-14 × RNOYR-17 & Erect & Low & Green & Smooth & Green & Green \\
\hline RNOYR-14 × RNOYR-18 & Erect & Low & Green & Smooth & Green & Green \\
\hline RNOYR-14 × RNOYR-24 & Erect & Low & Green & Smooth & Green & Green \\
\hline RNOYR-15 × RNOYR-16 & Erect & Low & Green with purple tinge & Smooth & Green & Green \\
\hline RNOYR-15 × RNOYR-17 & Erect & Low & Green with purple tinge & Slightly rough & Green & Green \\
\hline RNOYR-15 × RNOYR-18 & Erect & Low & Green with purple tinge & Slightly rough & Green & Green \\
\hline RNOYR-15 × RNOYR-24 & Erect & Low & Green with purple tinge & Slightly rough & Green & Green \\
\hline RNOYR-16 × RNOYR-17 & Erect & Low & Green with purple tinge & Smooth & Green & Green \\
\hline RNOYR-16 × RNOYR-18 & Erect & Low & Green & Slightly rough & Green & Green \\
\hline RNOYR-16 × RNOYR-24 & Erect & Low & Green & Smooth & Green & Green \\
\hline RNOYR-17 × RNOYR-18 & Erect & Low & Green with purple tinge & Slightly rough & Green & Green \\
\hline RNOYR-17 × RNOYR-24 & Erect & Low & Green with purple tinge & Smooth & Green & Green \\
\hline RNOYR-18 × RNOYR-24 & Erect & Low & Green with purple tinge & Smooth & Green & Green \\
\hline \multicolumn{7}{|l|}{ Hybrid checks } \\
\hline No. 64 & Erect & Low & Green & Smooth & Green & Green \\
\hline Avantika & Erect & Low & Green & Smooth & Green & Green \\
\hline Shakti & Erect & Low & Green & Smooth & Green & Green \\
\hline \multicolumn{7}{|l|}{ OPV checks } \\
\hline Pusa Sawani & Erect & Low & Green with purple tinge & Smooth & Green & Green \\
\hline Arka Anamika & Erect & Low & Green with purple tinge & Smooth & Green & Green \\
\hline
\end{tabular}

(b)

\begin{tabular}{|c|c|c|c|c|c|c|}
\hline$F_{1}$ hybrid & Leaf lobing & $\begin{array}{c}\text { Leaf } \\
\text { pubescence }\end{array}$ & Petiole colour & $\begin{array}{l}\text { Petal } \\
\text { colour }\end{array}$ & $\begin{array}{c}\text { Outside } \\
\text { petal base } \\
\text { colour }\end{array}$ & $\begin{array}{c}\text { Shape of } \\
\text { epicalyx } \\
\text { segments }\end{array}$ \\
\hline \multicolumn{7}{|l|}{ Single cross hybrids } \\
\hline RNOYR-14 × RNOYR-15 & Deeply lobed & Slightly rough & Green with red colour & Yellow & Red & Lanceolate \\
\hline RNOYR-14 × RNOYR-16 & Deeply lobed & Downy & Green with red colour & Yellow & Red & Lanceolate \\
\hline RNOYR-14 × RNOYR-17 & Deeply lobed & Downy & Green with red colour & Yellow & Red & Lanceolate \\
\hline RNOYR-14 × RNOYR-18 & Shallowly lobed & Slightly rough & Green with red colour & Yellow & Red & Lanceolate \\
\hline RNOYR-14 × RNOYR-24 & Deeply lobed & Downy & Green & Yellow & Yellow & Lanceolate \\
\hline RNOYR-15 × RNOYR-16 & Deeply lobed & Downy & Green with red colour & Yellow & Red & Lanceolate \\
\hline
\end{tabular}




\section{Continued}

\begin{tabular}{lcccccc}
\hline RNOYR-15 $\times$ RNOYR-17 & Deeply lobed & Slightly rough & Green with red colour & Yellow & Red & Lanceolate \\
RNOYR-15 $\times$ RNOYR-18 & Deeply lobed & Slightly rough & Green with red colour & Yellow & Red & Lanceolate \\
RNOYR-15 $\times$ RNOYR-24 & Deeply lobed & Slightly rough & Green with red colour & Yellow & Red & Lanceolate \\
RNOYR-16 $\times$ RNOYR-17 & Deeply lobed & Downy & Green with red colour & Yellow & Red & Lanceolate \\
RNOYR-16 $\times$ RNOYR-18 & Shallowly lobed & Slightly rough & Green with red colour & Yellow & Red & Lanceolate \\
RNOYR-16 $\times$ RNOYR-24 & Deeply lobed & Downy & Green & Yellow & Yellow & Lanceolate \\
RNOYR-17 $\times$ RNOYR-18 & Deeply lobed & Slightly rough & Green with red colour & Yellow & Red & Lanceolate \\
RNOYR-17 $\times$ RNOYR-24 & Deeply lobed & Slightly rough & Green with red colour & Yellow & Red & Lanceolate \\
RNOYR-18 $\times$ RNOYR-24 & Shallowly lobed & Slightly rough & Green with red colour & Yellow & Red & Lanceolate \\
Hybrid checks & & & & & & \\
No. 64 & Deeply lobed & Slightly rough & Green with red colour & Yellow & Red & Lanceolate \\
Avantika & Deeply lobed & Slightly rough & Green with red colour & Yellow & Red & Lanceolate \\
Shakti & Deeply lobed & Slightly rough & Green with red colour & Yellow & Red & Lanceolate \\
OPV checks & & & & & Red & Lanceolate \\
Pusa Sawani & Shallowly lobed & Downy & Green with red colour & Yellow & Red \\
Arka Anamika & Shallowly lobed & Downy & Green with red colour & Yellow & Red & Lanceolate \\
\hline
\end{tabular}

(c)

\begin{tabular}{|c|c|c|c|c|c|c|}
\hline$F_{1}$ hybrid & $\begin{array}{c}\text { Position of fruit } \\
\text { on stem }\end{array}$ & Fruit shape & $\begin{array}{l}\text { Immature fruit } \\
\text { colour }\end{array}$ & $\begin{array}{c}\text { Fruit } \\
\text { pubescence }\end{array}$ & $\begin{array}{l}\text { Number } \\
\text { of ridges } \\
\text { per fruit }\end{array}$ & $\begin{array}{l}\text { Protrusion of seeds } \\
\text { through epidermis }\end{array}$ \\
\hline \multicolumn{7}{|l|}{ Single cross hybrids } \\
\hline RNOYR-14 × RNOYR-15 & Semi erect & Angular & Green & Downy & 5 & Absent \\
\hline RNOYR-14 × RNOYR-16 & Erect & Angular & Green & Downy & 5 & Absent \\
\hline RNOYR-14 × RNOYR-17 & Erect & Angular & Green & Downy & 5 & Absent \\
\hline RNOYR-14 × RNOYR-18 & Erect & Angular & Green & Downy & 5 & Absent \\
\hline RNOYR-14 × RNOYR-24 & Erect & Angular & Green & Downy & 5 & Absent \\
\hline RNOYR-15 × RNOYR-16 & Erect & Angular & Green & Downy & 5 & Absent \\
\hline RNOYR-15 × RNOYR-17 & Erect & Angular & Green & Downy & 5 & Absent \\
\hline RNOYR-15 × RNOYR-18 & Semi erect & Angular & Green & Downy & 5 & Absent \\
\hline RNOYR-15 × RNOYR-24 & Erect & Angular & Green & Downy & 5 & Absent \\
\hline RNOYR-16 × RNOYR-17 & Erect & Angular & Green & Downy & 5 & Absent \\
\hline RNOYR-16 × RNOYR-18 & Erect & Angular & Green & Downy & 5 & Absent \\
\hline RNOYR-16 × RNOYR-24 & Erect & Angular & Green & Downy & 5 & Absent \\
\hline RNOYR-17 × RNOYR-18 & Erect & Angular & Dark green & Downy & 5 & Absent \\
\hline RNOYR-17 × RNOYR-24 & Erect & Angular & Green & Downy & 5 & Absent \\
\hline RNOYR-18 × RNOYR-24 & Erect & Angular & Green & Downy & 5 & Absent \\
\hline \multicolumn{7}{|l|}{ Hybrid checks } \\
\hline No. 64 & Erect & Angular & Green & Downy & 5 & Absent \\
\hline Avantika & Erect & Angular & Green & Downy & 5 & Absent \\
\hline Shakti & Erect & Angular & Green & Downy & 5 & Absent \\
\hline \multicolumn{7}{|l|}{ OPV checks } \\
\hline Pusa Sawani & Erect & Angular & Green & Downy & 5 & Absent \\
\hline Arka Anamika & Erect & Angular & Green & Downy & 5 & Absent \\
\hline
\end{tabular}

OPV = Open pollinate variety. 
leaf pubescence (downy and slightly rough), separately. Hybrids were grouped into two groups on the basis of their stem colour (green and green with purple tinge) and stem pubescence (smooth and slightly rough), separately. Although, there were no differences in petal color, clear cut difference was found in outside petal base colour (red and yellow) among the hybrids. There was no difference in shape of epicalyx segments (only lanceolate) among the hybrids. The fruits of all hybrids under study had erect position on main stem except RNOYR-14 × RNOYR-15 and RNOYR-15 × RNOYR-18 (semi-erect), downy pubescence and green immature fruit colour except RNOYR-17 $\times$ RNOYR-18 (dark green), five ridges and angular shape.

The variation in leaf shape, leaf pubescence, stem colour, stem pubescence, petiole colour, outside petal base colour, immature fruit colour and position of fruit on main stem, were easily recognizable with visual appraisal in the material. Fruits displayed diversity in colour (green/dark green) and its position on main stem (erect/ semi-erect). Fruits with characteristics such as smooth, spineless, slender with green (green/dark green) skin are very desirable in the Indian local and export markets. RNOYR-15, RNOYR-17 and RNOYR-18 were among the parental lines and RNOYR-17 $\times$ RNOYR-18 was among the crosses that had dark green fruits. In the present study, the comparative view of fruits of six parental lines, their fifteen crosses and three hybrid checks is depicted in Figure 1. In the present study, all of the 15 hybrids and three hybrid checks under present study were of superior pod quality as evident from the qualitative traits (Table 5 and Figure 1).

\subsection{Field Screening of Single Cross Hybrids to YVMV}

Field screening under natural epiphytotic conditions during summer at Hyderabad indicated that none of the15 single cross hybrids and their 6 parental inbred lines had any incidence of YVMV ( $0 \%$ incidence) on plants at final harvest (Table 4). Results of this field screening under hot spot area indicated that all of the 15 hybrids and
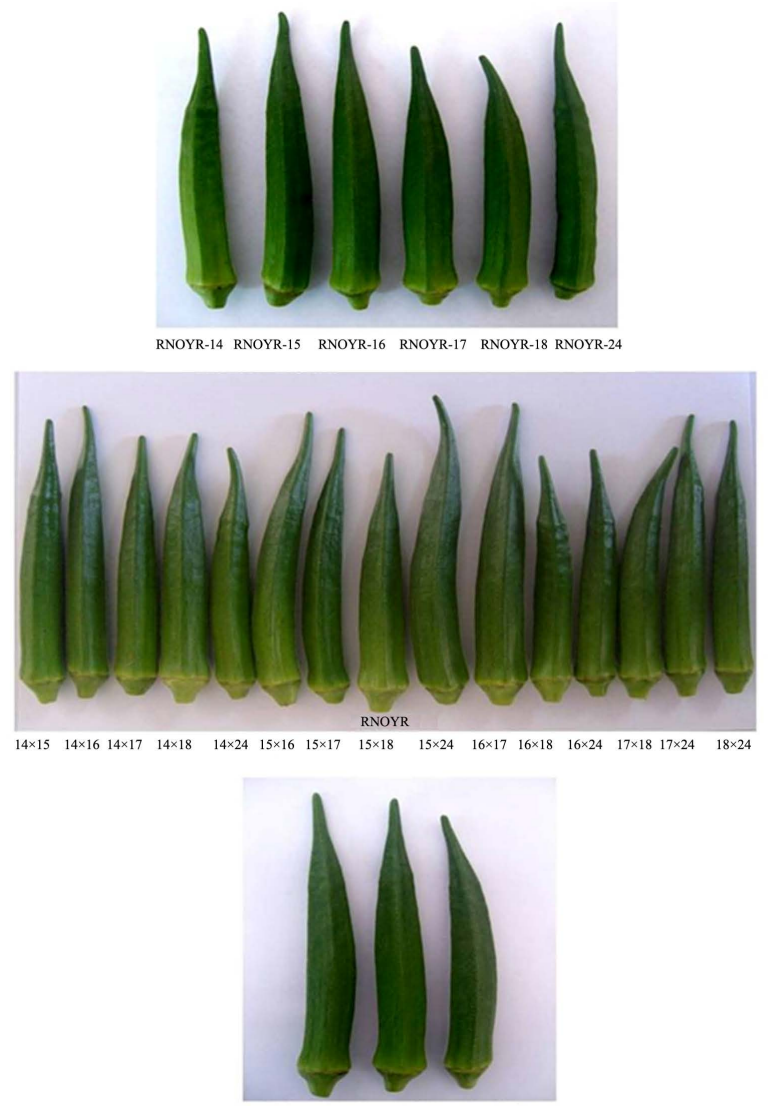

No. 64 Avantika Shakti

Figure 1. Comparative view of pods of parental inbred lines (top row), single cross hybrids (middle row) and hybrid checks (bottom row) of okra at horticultural maturity stage. 
their 6 parental inbred lines under study were found to be resistant to YVMV under field conditions. Of the three hybrid checks under study, "No. 64" was found to be resistant, while "Avantika" and "Shakti" were found to be tolerant. Both of the two susceptible checks viz., "Arka Anamika” and "Pusa Sawani” were found to be highly susceptible with 100\% YVMV incidence on plants.

\section{Discussion}

\subsection{Horticultural Superiority of Single Cross Hybrids}

To turn okra into a perfect candidate for sustainable agriculture, the cultivated variety should be attractive to both producers and consumers in terms of yield and quality (horticultural superiority), respectively [20]. A horticulturally superior vegetable variety requires several characteristics valued by consumers and providing economic sustainability for producers, every vegetable breeder faces the challenge of simultaneously selecting for "agro-economic" and "quality" traits. What can vegetable breeders do to assess the "agro-economic" and "quality" of their varieties?. When new hybrid varieties have been developed, they should be evaluated in variety trials based on certain "agro-economic" and "quality" traits. Agro-economic traits that an okra breeder must select for include biomass productivity and traditional yield components such as number and size of pods. Consumer purchases of okra are enthused based on "quality" traits inherent to the pods such as size, shape and color. Thus, yield is partly about producing tonnage, but also about the proportion of the crop that can be harvested and brought to market in a condition and at a price acceptable to the consumer.

\subsubsection{Yield Traits of Single Cross Hybrids}

In general, the significant differences $(P \leq 0.05)$ revealed among the agro-economic traits may be due to environmental influences on the genotypes as well as differences in the genetic potential of the different okra genotypes (parental lines, crosses, hybrid checks and OPV checks). This corroborates findings of earlier researchers [25] [26] who mentioned the role of environmental factors as well as differences in the genetic makeup of different varieties in yield determination of okra. Non-significant differences observed within replications suggested that the genotypes have enough variability for almost all the traits to carry out further genotypic studies. The non-significant differences observed in the days to $50 \%$ flowering indicate that the genetic components of the parental material are intact. It follows, therefore, that any improvement sort must be directed at the other characters except days to $50 \%$ flowering.

In okra, of the thirteen agro-economic traits under study, high mean values are desirable for plant height, number of branches per plant, fruit length, fruit width and fruit weight, total number of fruits per plant, number of marketable fruits per plant, total yield per plant and marketable yield per plant, while low mean values are desirable for internodal length, days to 50\% flowering, first flowering node and first fruiting node. Plant height, number of branches per plant and internodal length largely determine the fruit bearing surface and were considered as important growth attributes. Okra bears pods at almost all nodes on main stem. Higher the plant height, higher is the number of fruits per plant because of accommodation of more number of nodes for a given internodal length. Higher the number of branches on the main stem, higher is the number of fruits per plant because of accommodation of more number of nodes per plant. Shorter distance between nodes accommodates more number of nodes on main stem, which will ultimately lead to higher fruit number and higher fruit production. Hence, high mean value is desirable for plant height and number of branches per plant, while low mean value is desirable for internodal length to accommodate more number of nodes and to get higher fruit yield in okra. Days to $50 \%$ flowering, first flowering node and first fruiting node are the indicators of earliness in okra. Earliness is one of the main objectives of okra breeding. Earliness, expressed by the lower leaf axils in which flower buds appear is partly due to varietal characteristic. It allows completion of pickings before the crop suffers damages from unfavorable climatic conditions. Early flowering not only gives early pickings and better returns but also widens fruiting period of the plant. In addition, it also reduces the losses due to the attack of insects and diseases. Days to $50 \%$ flowering are of importance in the sense that if flowering starts earlier, sufficient time will be available for flowering and fruit formation process. Thus, early flowering is desirable in okra and the genotypes with early flowering habit are desirable. Flowering and fruiting at lower nodes are helpful in increasing the number of fruits per plant as well as getting early yields. Days to $50 \%$ flowering, first flowering node and first fruiting node are the effective traits for earliness. Low mean value is highly desirable for all these three attributes of earliness. Fruit length, width, weight and number are considered to be the fruit traits in okra, for 
which high mean value is desirable.

Optimizing pod yield is one of the most important goals for most okra growers and, consequently, most okra breeding programs [12]. For improving this crop through conventional breeding and selection, adequate knowledge of association that exists between yield and yield related characters is essential for the identification of selection procedure. Correlation coefficient analysis revealed that plant height, fruit length, fruit width, fruit weight, total number of fruits per plant, number of marketable fruits per plant and total yield per plant had significant positive correlation, while number of branches per plant, internodal length, days to $50 \%$ flowering, first flowering node and first fruiting node had significant negative correlation with marketable yield per plant [12]. From the perusal of mean performance of single cross hybrids, it is evident that the hybrids showing high mean values for marketable yield per plant might have been due to their high mean values for total yield per plant, total number of fruits per plant, number of marketable yield per plant, fruit length, width and weight, plant height and number of branches per plant and low mean values for internodal length, days to $50 \%$ flowering, first flowering and fruiting nodes, which could be some of the yield contributing characters under study. In the present study, on the basis of mean performance for both total and marketable yield per plant and the associated yield attributes, the crosses RNOYR-15 × RNOYR-16, RNOYR-16 × RNOYR-17 and RNOYR-17 × RNOYR-18 were the top three per se performers, which could be exploited either in breeding programs to generate genetic variability in desirable direction for effective selection to improve the respective traits or for commercial cultivation.

\subsubsection{Qualitative Traits of Single Cross Hybrids}

Variation is an important attribute in breeding programs [27] [28]. The okra entries characterized in this study showed a broad variation for most traits, which allows for the identification of promising parents and hybrids of okra. All of the fifteen crosses and their six parents, three hybrid checks and two OPV checks had the commonest growth habit i.e. indeterminate growth habit with erect general growth appearance (unique orthotrop axis). Erect plant type is advantageous to okra production since it would allow maximum and uniform exposure or distribution of all leaves and other vegetative parts for better interception of sunlight. This would result in an increase in dry matter production and a subsequent increase in yield. This is in conformity with the findings of earlier researcher [29] in tomato. Moreover, there is less chance of fruits touching the ground or soil thereby causing fruit rot. The variation in leaf shape, leaf pubescence, stem colour, stem pubescence, petiole colour, outside petal base colour, immature fruit colour and position of fruit on main stem, were easily recognizable with visual appraisal in the material. This could be due to the fact that the parental lines used in this study were improved lines and their crosses and hence less variable or diverse with respect to qualitative traits. Fruits with characteristics such as smooth, spineless, slender with green (green/dark green) skin are very desirable in the Indian local and export markets. RNOYR-15, RNOYR-17 and RNOYR-18 were among the parental lines and RNOYR-17 $\times$ RNOYR-18 was among the crosses that had dark green fruits.

Among the eighteen qualitative characters studied, there exists relationship between the stem colour of the accessions and the fruit colour as well as their petiole colour. The relationship was stronger between the stem colour and the fruit colour than between the stem colour and petiole colour of the accessions. The same trend was observed between the stem pubescence and the fruit pubescence. These may probably be as a result of one gene controlling the pair of traits of stem and fruit colour and stem and fruit pubescence in the various accessions. This was also reported by other researcher [30] who investigated variation in okra species and found that a large number of okra characters such as pigment colour and spines on the fruit surfaces are inherited in a simple fashion, suggesting that these characters are controlled by relatively few genes. For the leaf and petal colour, the genes controlling these traits are probably dominant genes as no variation was expressed amongst the crosses. On the basis of mean performance for both total and marketable yield per plant and fruit quality, the crosses RNOYR-15 $\times$ RNOYR-16, RNOYR-16 $\times$ RNOYR-17 and RNOYR-17 $\times$ RNOYR-18 were the top three per se performers, which are statistically comparable with three hybrid checks under study.

\subsubsection{Pod Quality of Single Cross Hybrids}

Vegetable product innovation is necessary for maintaining the interest of today's consumer. Quality in vegetable crops, in contrast to field crops, is often more important than yield. For farmers to survive, varieties must be accepted by the market. Thus, quality is usually more important than productivity. The final goal of vegetable breeding programs is then to release new varieties having elite combinations of many desirable horticultural 
characteristics. In addition to pod yield, pod quality plays an important role in okra productivity and marketability [31]. The economic returns from okra not only depend on pod yield, but also on its quality, which is conglomerate of several horticultural traits [18]. In fruit vegetables like okra, the pod quality characteristics include size (length and width), shape, color, straightness, ribbedness, pubescence (texture), longitudinal groves (furrows) and constriction at base. These are the external qualities or properties on the basis of which consumers may be attracted to okra pods with greater perceived quality. Many local, open pollinated and hybrid varieties of okra occur in India, which differ from each other in fruit characteristics like size (length: short/long, width: thin/ broad or slender/wide), shape (round/angular/star shaped), colour (light green/dark green/red), ribbedness (5 ridged/multi-ridged (>10 ridges), straightness (straight/curved), pubescence (hairy/glabrous), longitudinal groves (furrows) (absent/present), constriction at base (absent/present). In quality breeding programs of okra for domestic markets for fresh consumption, the qualitative traits that need to be given emphasis include medium long to short, green, smooth (downy), five ridged, angular, straight fruits with blunt tip for enhanced fruit quality and appearance [18]. Several desirable quality characteristics of okra fruit are length, diameter, greenness, mucilage and fiber content. Industry and commercial preference is oriented to fruits $8.9-12.7 \mathrm{~cm}$ long (medium), although smaller fruits are commonly accepted in USA. A specific greenness and fruit diameter are also required, but they are often arbitrarily indicated (e.g. very dark and reduced fruit diameter are the most preferred) [31]. Greenness, length and weight of the fruit were associated with okra genotype [32]. Hence, trading in okra is done according to its quality and size.

One important factor in producing quality okra pods is the selection of superior varieties. Although $\mathrm{F}_{1}$ hybrids are not perfect, they are generally superior in quality and should be chosen to fit our specific needs. In the present study, all of the 15 hybrids and three hybrid checks under present study were of superior pod quality as evident from the qualitative traits. The findings of this study are important by their relevance to both, consumer preferences and the needs of genetic improvement in okra. On the whole, most of these hybrids under present study would be advantageous for quality improvement, trade facilitation and consumer protection.

There is potential of increased pod yields through use of $F_{1}$ hybrids in okra. On the basis of mean performance for pod yield and its components and acceptable pod quality traits, three crosses RNOYR-15 $\times$ RNOYR16, RNOYR-6 $\times$ RNOYR-17 and RNOYR-17 $\times$ RNOYR-18 were of statistically higher yield potential than the check "No. 64" and of statistically comparable yield potential with the hybrid checks "Avantika" and "Shakti". However, for more conclusive results there is need to subject the $F_{1}$ hybrids to the other two growing seasons and regions of the state.

\subsection{Disease Reaction of Single Cross Hybrids to YVMV}

Plant diseases continue to play a major limiting role in agricultural production. The control of plant diseases using classical pesticides raises serious concerns about food safety, environmental quality and pesticide resistance, which have dictated the need for alternative pest management techniques. It is important to find alternative measures to control plant diseases which do not harm the environment and at the same time increase yield and improve product quality [33]-[35]. Plant disease resistance and tolerance are genetically controlled [36]. In well-managed farming systems, crop losses to diseases can often be kept to an acceptable minimum by deploying resistant varieties. The development of varieties resistant to common, crop specific pathogens of economic importance is essential to reducing the pesticides needed and the energy consumed by low-input, medium-input and high-input farming systems to improve yield. A resistant variety can provide a base on which to construct an integrated control system [37] [38] and may be most fruitful when used in connection with other methods of control.

Viruses can substantially reduce production and quality and are becoming increasingly problematic worldwide due to the absence of virus resistant germplasm for many important vegetable crops. Viruses pose serious constraints to okra production. Okra is susceptible to at least 19 plant viruses [39]. These viruses severely affect okra production in terms of yield and fruit quality. Among these viruses, YVMV transmitted by whiteflies, is the most pressing plant protection problem universally faced by all okra growers [40] [41] causes significant losses in the okra production. Some attempts had been made by several workers to reduce to disease through the vector control [42]-[45] and also through the breeding strategies [46] [47]. Resistance breeding for YVMV to develop YVMV resistant variety is essential for stable and sustainable okra production.

Selecting $F_{1}$ hybrids and disease resistant cultivars cannot be stressed enough, in choosing wisely, farmers 
avoid many disease problems in the field. In okra, the easiest method of screening genotypes against YVMV is the field screening in the hotspot regions. In screening okra genotypes to YVMV, selection of the screening region as hot-spot is most important. Once the hot-spot region is selected, the selection of suitable screening season is also important. The role of environmental conditions cannot be denied in case of creation of epiphytotic situations in YVMV disease of okra. Each parameter of environment plays its role in reducing or enhancing of pathogenic activity as well as vectors especially insect vectors. Hot weather with little or no rainfall was conducive for disease progress of YVMV and also for multiplication of Bemisia tabaci [48]. Rise in minimum temperature is conducive for disease development while increase in relative humidity is detrimental to whitefly population [49]. In general, favorable environmental factors for the occurrence of YVMV in okra are high temperature and low and/or high humidity. Of the three regular growing seasons found in Andhra Pradesh viz., rainy season (June-September), winter season (October-January) and summer season (February-May), YVMV incidence is highest in summer season, lowest in winter season and moderate in rainy season in a given set of genotypes of okra screened under field conditions at Hyderabad (unpublished data). The climatic conditions of Hyderabad, Andhra Pradesh, India are high temperature and low humidity during summer. Hence, Hyderabad is one of the hot-spots for field screening for YVMV resistance in okra. Natural inoculum was relied upon for infection of YVMV.

Results of the field screening under hot spot area indicated zero percent incidence of YVMV in all of the 15 hybrids and their 6 parental inbred lines, while 100 percent incidence of YVMV in the two OPV checks "Pusa Sawani" and "Arka Anamika" used as cross-checks at final harvest. All of the 15 hybrids and their 6 parental inbred lines under study were found to be resistant to YVMV under field conditions. Of the three hybrid checks under study, "No. 64" was found resistant, while "Avantika" and "Shakti" were tolerant to YVMV. These could be useful sources of resistant genes which could be exploited in breeding okra cultivars resistant to YVMV. It was evident from the results of the present study that tolerance/resistance in okra is genetic and not due to escape. Of the 51 okra hybrids screened for YVMV resistance four hybrids were highly resistant to YVMV, while the rest of the hybrids were susceptible or highly susceptible [50]. The incidence of YVMV was higher during the rainy season when six varieties of okra were screened for resistance to YVMV in the rainy and summer seasons [51]. The incidence of YVMV was higher during the rainy season.

\section{Conclusion}

In conclusion, the analysis of variance revealed a wide range of variation for almost all the characters studied. Further, the present study revealed horticultural superiority and YVMV resistance of certain single cross hybrids over hybrid checks. The single cross hybrids RNOYR-15 $\times$ RNOYR-16, RNOYR-16 $\times$ RNOYR-17 and RNOYR-17 $\times$ RNOYR-18 with high yield potential, good fruit quality and YVMV resistance have got the potential of being commercially exploited for the production of $F_{1}$ hybrids in okra after further multi-environment testing. These hybrids would be advantageous for production and quality improvement, trade facilitation and environmental protection. The results of this study would help okra breeders to develop not only productive varieties but also those with YVMV resistance and superior pod quality attributes. In perspective, it would be judicious to produce $F_{1}$ hybrids and to evaluate their yield potential and pod quality. This could probably the first report of developing horticulturally superior and YVMV resistant single cross hybrids of okra in the world.

\section{References}

[1] Purseglove, J.W. (1987) Tropical Crops: Dicotyledons. Longman Singapore Publishers (Pvt.) Ltd., Singapore.

[2] Sinnadurai, S. (1992) Vegetable Production in Ghana. Asempa Publishers Ltd., Accra, 208.

[3] Saifullah, M. and Rabbani, M.G. (2009) Evaluation and Characterization of Okra (Abelmoschus esculentus L. Moench.) Genotypes. SAARC Journal of Agriculture, 7, 92-99.

[4] Reddy, M.T. (2010) Genetic Diversity, Heterosis Combining Ability and Stability in Okra (Abelmoschus esculentus (L) Moench). Ph.D. Thesis, Acharya N. G. Ranga Agricultural University, Hyderabad, 313.

[5] Martin, F.W. (1982) A Second Edible Okra Species and Its Hybrid with Common Okra. Annals of Botany, 50, 277283.

[6] Markose, B.L. and Peter, K.V. (1990) Okra. In: Review of Research on Vegetable and Tuber Crops, Technical Bulletin 16, Kerala Agricultural University Press, Mannuthy, Kerala, 109.

[7] Preston, S.R. (1998) Aibika/Bele-Abelmoschus manihot (L.) Medik. International Plant Genetic Resources Institute 
(IPGRI) Publication, Rome, IT, 97.

[8] Schippers, R.R. (2000) African Indigenous Vegetable an Overview of the Cultivated Species. National Resources Institute (NRI), University of Greenwich, London, 214.

[9] Duzyaman, E. (1997) Okra, Botany and Horticulture. Horticulture Reviews, 29, 41-72. http://dx.doi.org/10.1002/9780470650660.ch2

[10] Qhureshi, Z. (2007) Breeding Investigation in Bhendi (Abelmoschus esculentus (L.) Moench). M.Sc. Thesis, University of Agriculture Sciences, GKVK, Bangalore.

[11] Reddy, M.T., Haribabu, K., Ganesh, M., Begum, H., Babu, J.D. and Reddy, R.V.S.K. (2013) Gene Action and Combining Ability of Yield and Its Components for Late kharif Season in Okra (Abelmoschus esculentus (L.) Moench). Chilean Journal of Agricultural Research, 73, 9-16. http://dx.doi.org/10.4067/S0718-58392013000100002

[12] Reddy, M.T., Haribabu, K., Ganesh, M., Reddy, K.C., Begum, H., Reddy, R.V.S.K. and Babu, J.D. (2013) Correlation and Path Coefficient Analysis of Quantitative Characters in Okra (Abelmoschus esculentus (L.) Moench). Songklanakarin Journal of Science and Technology, 35, 243-250.

[13] Reddy, M.T., Haribabu, K., Ganesh, M. and Begum, H. (2012) Heterosis for Yield and Yield Components in Okra (Abelmoschus esculentus (L.) Moench). Chilean Journal of Agricultural Research, 72, 316-324. http://dx.doi.org/10.4067/S0718-58392012000300003

[14] USAID (2006) Activite De Renforcement De La Commercialisation Agricole En Guinee, Lafilieregombo (Okra) en Guinee, 32.

[15] Thakur, M.R. (1976) Inheritance of Resistance to Yellow Vein Mosaic (YVM) in a Cross of Okra Species, Abelmoschus esculentus and A. manihot ssp. Manihot. SABRAO Journal, 8, 69-73.

[16] Nerkar, Y.S. and Jambhale, N.D. (1985) Transfer of Resistance to Yellow Vein Mosaic from Related Species into Okra (Abelmoschus esculentus (L.) Moench). Indian Journal of Genetics and Plant Breeding, 45, 261-270.

[17] NHB (2013) Indian Horticulture Database 2013. Ministry of Agriculture, Government of India, Gurgaon, 289.

[18] Reddy, M.T., Haribabu, K., Ganesh, M., Reddy, K.C., Begum, H., Reddy, B.P. and Narshimulu, G. (2012) Genetic Variability Analysis for the Selection of Elite Genotypes Based on Pod Yield and Quality from the Germplasm of Okra (Abelmoschus esculentus L. Moench). Journal of Agricultural Technology, 8, 639-655.

[19] Ezeaku, I.E. and Awopetu, J.A. (1992) A Numerical Analysis of Variation in Some Accessions in Soyabeans. Nigerian Journal of Botany, 5, 51-61.

[20] Reddy, M.T., Haribabu, K., Ganesh, M., Begum, H. and Reddy, R.V.S.K. (2012b) Exploitation of Heterosis for Growth, Earliness and Yield Attributes in Okra (Abelmoschus esculentus (L) Moench). International Journal of Plant Breeding, 6, 53-60.

[21] Mahajan, R.K., Sapra, R.L., Srivastava, U., Singh, M. and Sharma, G.D. (2000) Minimal Descriptors (for Characterization and Evaluation) of Agri-Horticultural Crops (Part-I). National Bureau of Plant Genetic Resources, Pusa Campus, New Delhi, 230.

[22] United States Department of Agriculture (USDA) (1997) Agricultural Marketing Service, Fruit and Vegetable Division. 1965. Reprinted January 1997. United States Standards for Grades of Okra for Processing.

[23] Panse, V.G. and Sukhatme, P.V. (1985) Statistical Methods for Agricultural Workers. Indian Council of Agricultural Research Publication, 87-89.

[24] Sharma, B.R. and Sharma, O.P. (1984) Field Evaluation of Okra Germplasm against Yellow Vein Mosaic Virus. Punjab Horticulture Journal, 24, 131-133.

[25] Ariyo, O.J. (1993) Genetic Diversity in West African Okra (Abelmoschus caillei (A. Chev.) Stevels) Multivariate Analysis of Morphological and Agronomic Characteristics. Genetic Resources and Crop Evolution, 40, 25-32. http://dx.doi.org/10.1007/BF00053461

[26] Adeniji, O.T. (2003) Inheritance Studies in West African Okra (A. caillei). MSc Thesis, University of Agriculture, Abeokuta, 98.

[27] Hazra, P. and Basu, D. (2000) Genetic Variability, Correlation and Path Analysis in Okra. Annals of Agricultural Research, 21, 452-453.

[28] Omonhinmin, C.A. and Osawaru, M.E. (2005) Morphological Characterization of Two Species of Abelmoschus, Abelmoschus esculentus and Abelmoschus caillei. Plant Genetic Recourses Newsletter, 144, 51-55.

[29] Hanson, P. (2005) Lecture Notes on Tomato Breeding. Asian Vegetable Research and Development Center, Africa Regional Program Training, Arusha, Tanzania, 14.

[30] Akinyele, B.O. and Osekita, O.S. (2006) Correlation and Path Coefficient Analyses of Seed Yield Attributes in Okra (Abelmoschus esculentus (L) Moench). African Journal of Biotechnology, 5, 1330-1336. 
[31] Diaz Franco, A., Ortegon Morales, A. and Loera Gallardo, J. (1997) Fruit Characteristics and Yield of New Okra Hybrids. Subtropical Plant Science, 49, 8-11.

[32] Martin, F.W., Rhodes, A., Ortiz, M. and Diaz, F. (1981) Variation in Okra. Euphytica, 30, 697-705. http://dx.doi.org/10.1007/BF00038798

[33] Atkinson, D. and McKinlay, R.G. (1997) Crop Protection and Its Integration within Sustainable Farming Systems. Agriculture, Ecosystems \& Environment, 64, 87-93. http://dx.doi.org/10.1016/S0167-8809(97)00026-1

[34] Batish, D.R., Singh, H.P., Setia, N., Kohli, R.K., Kaur, S. and Yadav, S.S. (2007) Alternative Control of Little Seed Canary Grass Using Eucalypt Oil. Agronomy for Sustainable Development, 27, 171-177. http://dx.doi.org/10.1051/agro:2007008

[35] Camprubi, A., Estaun, V., El Bakali, M.A., Garcia-Figueres, F. and Calvet, C. (2007) Alternative Strawberry Production Using Solarization, Metham Sodium and Beneficial Soil Microbes as Plant Protection Methods. Agronomy for Sustainable Development, 27, 179-184. http://dx.doi.org/10.1051/agro:2007007

[36] Agrios, N.G. (2005) Plant Pathology. 5th Edition, Elsevier, Amsterdam, 635.

[37] Maxwell, F.G., Johnie, N.J. and Parrott, W.L. (1972) Resistance of Plant to Insects. Advances in Agronomy, 24, 187265. http://dx.doi.org/10.1016/S0065-2113(08)60636-7

[38] Gallun, R.L., Starks, K.J. and Guthrie, W.D. (1975) Plant Resistance to Insects Attacking Cereals. Annual Review of Entomology, 20, 337-357. http://dx.doi.org/10.1146/annurev.en.20.010175.002005

[39] Brunt, A., Crabtree, K. and Gibbs, A. (1990) Viruses of Tropical Plants. CAB International, Wallingford.

[40] Alegbejo, M., Ogunlana, M. and Banwo, O. (2008) Survey for Incidence of Okra Mosaic Virus in Northern Nigeria and Evidence for Its Transmission by Beetles. Spanish Journal of Agriculture Research, 6, 408-411. http://dx.doi.org/10.5424/sjar/2008063-342

[41] Benchasri, S. (2012) Screening for Yellow Vein Mosaic Virus Resistance and Yield Loss of Okra under Field Conditions in Southern Thailand. Journal of Animal and Plant Sciences, 12, 1676-1686.

[42] Shastry, K.S.M. and Singh, S.J. (1931) Restriction of Yellow Vein Mosaic Virus Spread in Okra through the Control of Vector Whitefly (Bemisia tabaci). Indian Journal of Mycology and Plant Pathology, 3, 76-80.

[43] Chakraborty, R. and Mukhopadhyay, S. (1977) Effect of Some Pesticides on the YVMV Disease of Bhindi. Pesticides, 11, 19-22.

[44] Singh, B.R. and Singh, M. (1989) Control of Yellow Vein Mosaic of Okra by Checking Its Vector Whitefly through Adjusting Dates of Sowing, Insecticidal Application and Crop Barrier. Indian Journal of Virology, 5, 61-66.

[45] Bhagat, A.P., Yadav, B.I. and Prasad, Y. (1997) Management of Bhindi Yellow Vein Mosaic Virus Disease by Insecticides. Journal of Mycology and Plant Pathology, 27, 215-216.

[46] Jambhale, N.D. and Nerkar, Y.S. (1981) Inheritance of Resistance to Okra Yellow Vein Mosaic Disease in Inter-Specific Crosses of Abelmoschus. Theoretical and Applied Genetics, 60, 313-316. http://dx.doi.org/10.1007/BF00263725

[47] Nerkar, Y.S. and Jambhale, N.D. (1986) Breeding Okra for Resistance to Yellow Vein Mosaic Virus. Indian Journal of Virology, 3, 94-98.

[48] Singh, J.S. (1990) Etiology and Epidemiology of Whitefly Transmitted Virus Disease of Okra. Indian Plant Disease Research, 5, 64-70.

[49] Ali, S., Khan, M.A., Habib, A., Rasheed, S. and Iftikhar, Y. (2005) Correlation of Environmental Conditions with Okra Yellow Vein Mosaic Virus and Bemisia tabaci Population Density. International Journal of Agriculture and Biology, 7, 142-144.

[50] Dhankhar, S.K., Dhankhar, B.S. and Saharan, B.S. (1996) Screening of Okra Genotypes for Resistance to Yellow Vein Mosaic Disease. Annals of Biology, 12, 90-92.

[51] Sangar, R.B.S. (1997) Field Reaction of Bhindi Varieties to Yellow Vein Mosaic Virus. Indian Journal of Virology, 13, 131-134. 